\title{
Prognostic COMPARISON OF THE PROLIFERATION MARKERS (MitoTiC ACTIVITY INDEX, PHOSPHOHisTONE H3, Ki67), STEROID RECEPTORS, HER2, HIGH MOLECULAR WEIGHT CYTOKERATINS AND CLASSICAL PROGNOSTIC FACTORS IN $\mathbf{T}_{1-2} \mathbf{N}_{0} \mathbf{M}_{0}$ BREAST CANCER
}

Einar Gudlaugsson ${ }^{1}$, Jan Klos ${ }^{1}$, Ivar Skaland ${ }^{1}$, Emiel A.M. Janssen ${ }^{1}$, Rune SmaAland ${ }^{2}$, Weiwei Feng ${ }^{3}$, Zhimin ShaO ${ }^{4}$, Anais Malpica 5 , Jan P.A. BaAK ${ }^{1,6}$

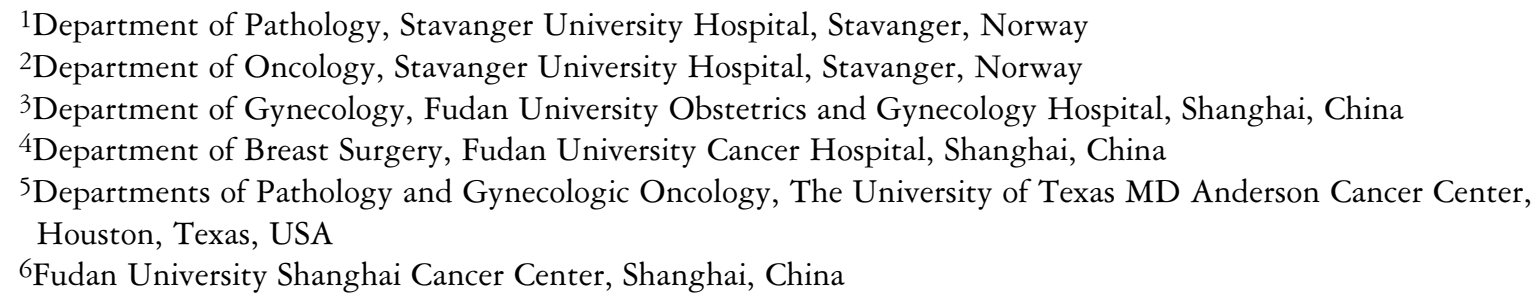

The proliferation factors: mitotic activity index (MAI), phosphohistone $\mathrm{H} 3(\mathrm{PPH} 3)$ and Ki67 have strong prognostic value in early breast cancer but their independent value to each other and other prognostic factors has not been evaluated.

In $237 \mathrm{~T}_{1-2} \mathrm{~N}_{0} \mathrm{M}_{0}$ breast cancers without systemic adjuvant treatment, formalized MAI assessment and strictly standardized, fully automated quantitative immunohistochemistry (IHC) for $\mathrm{Ki} 67, \mathrm{PPH} 3$, estrogen (ER) and progesterone receptor (PR), HER2, cytokeratins $-5 / 6$ and -14 , and automated digital image analysis (DIA) for measuring $\mathrm{PPH} 3$ and $\mathrm{Ki} 67$ were performed. Section thickness was measured to further control IHC measurements. All features were measured in the periphery of tumors. The different proliferation assessments and other well-established clinicopathological and biomarker prognostic factors were compared.

DIA-Ki67 added prognostically to PPH3. None of the other biomarkers or clinicopathological variables added prognostically to this $\mathrm{PPH} 3 / \mathrm{Ki} 67$ combination. However, when PPH3 is replaced by MAI the prognostic value is nearly the same.

In early operable node negative breast cancer without adjuvant systemic treatment, $\mathrm{Ki} 67$ with a threshold of $6.5 \%$ assessed by digital image analysis in the periphery of the tumor is prognostically strong. The combination of either PPH3/Ki67 or $\mathrm{MAI} / \mathrm{Ki} 67$ overshadowed the prognostic value of all other features including Ki67 alone.

Key words: breast cancer, proliferation, automation, Ki67, phosphohistone H3, mitosis. 


\section{Introduction}

Breast cancer is the most frequent female malignancy in the western world [1]. Treatment has developed considerably over the past decades. Several prognostic and predictive factors have been introduced to improve therapeutic decision-making $[2,3]$. Guidelines from Adjuvant!Online (AO), Sankt Gallen (SG) or the Norwegian Breast Cancer Group (NBCG) often combine conventional predictors to estimate relapse and mortality risk and classify the patients into low, intermediate, or high-risk groups [2]. Such factors identify $80 \%$ or more of all lymph node negative patients (LN-neg) as high risk, while only $15-20 \%$ of the patients die from metastatic disease when left untreated [4]. The use of these guidelines therefore means serious overtreatment.

Proliferation assessed by either mitotic activity index (MAI), phosphohistone $\mathrm{H} 3$ ( $\mathrm{PPH} 3$ ) or Ki67 has a strong prognostic value [5-7]. Lymph node negative breast cancer patients with a high proliferation index in general have a 3-6 times higher risk of dying from distant metastases than those with low proliferation [5]. Mitotic activity index has an accuracy in LN-neg considerably exceeding that of Adjuvant!Online and the Norwegian national breast cancer treatment guidelines and identifies patients who would have benefitted from adjuvant systemic treatment (AST), but were regarded as low-risk groups by Adjuvant! or the NBCG guidelines, and vice versa [8].

We have recently tested the reproducibility and prognostic value of different Ki67 measurement techniques which are widely used. The measurement techniques varied from interactive counts to fully automated image analysis. The results showed that counts of $\mathrm{Ki} 67$ positive cells by different pathologists were poorly reproducible. Interactive point-weighted counting of Ki67 by morphometric techniques were much more reproducible, but automated digital image analysis (DIA) was the most reproducible and prognostically strongest [9].

In the present study we compared, in operable node negative breast cancers of women aged less than 71 years without systemic adjuvant treatment, the prognostic value of the MAI, Ki67 and PPH3. For the MAI, formalized and strictly protocolized measurement was performed as described in the national Dutch MMMCP multicentre prospective evaluation. For Ki67 and PPH3, fully automated and standardized tissue processing, antigen retrieval and immuno-histochemical staining were done using strict standard operating procedures, while measurement was done by automated digital image analysis (using the previously established optimal prognostic threshold of $6.5 \%$ $\mathrm{Ki} 67$ and $\mathrm{PPH} 3$ positive cells). The results of the MAI, $\mathrm{PPH} 3$ and Ki67 were also compared with other wellestablished and validated prognosticators (estrogen and progesterone receptors (ER, PR), HER2 (neu) and cytokeratin-5/6).

\section{Material and methods}

\section{Patients}

The study was approved by the Regional Ethics Committee, the Norwegian Social Science Data Service, and the Norwegian Data Inspection. The results are presented in accordance with the reporting recommendations for tumor marker prognostic studies criteria [10].

Paraffin-embedded material from 384 consecutive invasive node negative breast cancer patients less than 71 years old with operable breast cancer treated between 1990 and 1997 from the Department of Pathology at the Stavanger University Hospital (Stavanger, Norway) was used. The following patients were excluded: patients who received adjuvant treatment $(\mathrm{n}=90)$, those with carcinoma in situ only or extensive carcinoma in situ with a small micro-invasive component $<0.5 \mathrm{~mm}$ that was ineligible for MAI evaluation $(\mathrm{n}=18)$, patients with recurrence within 6 months of follow-up $(\mathrm{n}=3)$, those with $<6$-month follow-up $(\mathrm{n}=5)$, and patients with Paget's disease $(\mathrm{n}=1)$, bilateral breast cancer $(n=4)$, or other previous malignancies $(n=2)$. Material was technically inadequate for 21 patients, leaving $240 \mathrm{~T}_{1-2} \mathrm{~N}_{0} \mathrm{M}_{0}$ patients for analysis. There was no significant difference in age or tumor size in the 240 patients when compared to the original $384 \mathrm{pa}-$ tients. All patients were treated with modified radical mastectomy $(\mathrm{n}=131)$ or breast-conserving therapy $(\mathrm{n}=109)$, always with adequate lymph node dissection (at least 10, median 13 nodes). Locoregional radiotherapy was administered to patients who underwent breast-conserving therapy or had medially localized tumors.

\section{Pathology}

The post-surgical size of the tumor was measured on the fresh specimens. Tumors were cut into $0.5 \mathrm{~cm}$ slices, fixed in $4 \%$ buffered formaldehyde, and embedded in paraffin. Paraffin sections were cut into highly standardized $4-\mu \mathrm{m}$ sections for hematoxylin-eosin (HE). Histological type was assessed according to World Health Organization criteria [11]. Grade (Grade $1=3,4$, or 5 ; Grade $2=6$ or 7; Grade $3=8$ or 9) was assessed according to the Nottingham modification [12], calculated as the sum of tubule formation $(>75 \%=1$, $10-75 \%=2$, and $<10 \%=3$ ), nuclear atypia (mild $=1$, moderate $=2$, and marked $=3$ ), and MAI class $(0-5=1,6-10=2$, and $>10=3)$.

\section{Mitotic activity index assessment}

The MAI was assessed as described in detail elsewhere [5, 8]. Briefly, all unambiguous mitoses were counted in 10 consecutive neighboring fields of vision (FOV) in the most cell-dense area $\left(1.59 \mathrm{~mm}^{2}\right.$ at specimen lev$\mathrm{el}$ ), usually in the periphery of the tumor (the so-called 
growing zone). For details of the counting method, see [7]. The MAI has been shown to be reproducible and insensitive to variations in tissue processing [13-15].

\section{Sections for immunohistochemistry}

Four-micrometer thick paraffin sections adjacent to the HE sections used for assessment of MAI, histology and immunohistochemistry (IHC) were mounted onto Superfrost Plus slides (Menzel, Braunschweig, Germany) and dried overnight at $37^{\circ} \mathrm{C}$ followed by $1 \mathrm{~h}$ at $60^{\circ} \mathrm{C}$. To ensure uniform handling of samples, all sections were made by the same person, on the same microtome with constant room temperature and constant rotation speed of the microtome, and processed simultaneously for IHC. We have shown before that the coefficient of variation of the section thickness is low and not a factor significantly influencing the prognostic value of $\mathrm{Ki} 67$ expression [9].

\section{Immunohistochemistry}

The immunohistochemical methods used have been carefully tested and compared with other methods to select the most optimal procedures, as described elsewhere [6]. In short, antigen retrieval and antibody dilution were optimized prior to the study onset. Sections were deparaffinized in xylene and rehydrated in decreasing concentrations of alcohol. Antigen was retrieved with a highly stabilized retrieval system (ImmunoPrep, Instrumec, Oslo, Norway) using $10 \mathrm{mM}$ TRIS/1 mM EDTA (pH 9.0) as the retrieval buffer. Sections were heated for $3 \mathrm{~min}$ at $110^{\circ} \mathrm{C}$ followed by $10 \mathrm{~min}$ at $95^{\circ} \mathrm{C}$ and cooled to $20^{\circ} \mathrm{C}$.

Rabbit polyclonal anti-phosphohistone $\mathrm{H} 3$ (ser 10) (Upstate \#06-570; Lake Placid, NY) was used at a dilution of 1 : 1500. Ki67 (clone MIB-1, DAKO, Glostrup, Denmark) was used at dilution $1: 100$. ER (clone SP1, Neomarkers/LabVision, Fremont, CA, USA) was used at a dilution of 1/400. PR (clone SP2, Neomarkers/LabVision) was used at a dilution of 1/1000.

For HER2 assessments, the HercepTest kit (DAKO) was used according to the manufacturer's FDA-approved procedures. HercepTest $2+$ and $3+$ cases were retested with the PathVysion (Vysis, Downers Grove, IL, USA) assay following the manufacturer's FDA-approved protocols. Only HER2 amplified cases were regarded as positive. Cytokeratin 5/6 (Clone D5/16 B4, Dako, Glostrup, Denmark) at a dilution of 1/100 and cytokeratin 14 (Clone LL002, Novocastra, Wetzlar, Germany) at a dilution of 1/40 were used. For lymph vessel invasion, the same protocol was used as described before [16]. Briefly, the sections were incubated with a primary antibody cocktail of p63 (Dako, Glostrup, Denmark, clone 4A4) and D2-40 (Dako, clone D2-40). The primary antibodies were diluted to a final dilution of $1: 1200$ and $1: 200$ respectively. In all protocols the Dako antibody diluent (S0809) was used.
Anti-phosphohistone $\mathrm{H} 3$ was incubated for $60 \mathrm{~min}$ at $22^{\circ} \mathrm{C}$. All other antibodies were incubated for $30 \mathrm{~min}$ at $22^{\circ} \mathrm{C}$. The EnVision ${ }^{\mathrm{TM}}$ Flex detection system (Dako, K8000) was used for visualization. Sections were incubated for $5 \mathrm{~min}$ with peroxidase-blocking reagent (SM801), 20 min with the EnVision ${ }^{\mathrm{TM}}$ FLEX/HRP Detection Reagent (SM802), 10 min with EnVisionTM FLEX DAB + Chromogen (DM827)/EnVision ${ }^{\mathrm{TM}}$ FLEX Substrate Buffer (SM803) mix and 5 min with EnVision TM FLEX Hematoxylin (K8008). The slides were then dehydrated and mounted. All immunohistochemical stainings were performed using a Dako Autostainer Link 48 instrument and EnVisionTM FLEX Wash Buffer (DM831).

Due to the small size of the invasive cancer left after recutting of the paraffin blocks, $\mathrm{Ki} 67$ could not be assessed in 3 cases, leaving 237 cases for analysis.

\section{Automated digital image analysis of $\mathrm{Ki} 67$ and $\mathrm{PPH} 3$}

We have described before how subjective counts and computerized interactive morphometry were done, but $\mathrm{Ki} 67$ and PPH3 expression assessment by the fully automated VIS digital image analysis (DIA) system (Visiopharm, Hørsholm, Denmark), using similar image processing principles as described before [6], was much more reproducible and also stronger prognostically [9]. Reference is made to that original detailed description and a brief treatise will follow here. Depending on the tumor diameter, two to ten square areas of each $1.59 \mathrm{~mm}^{2}$ with subjectively the highest $\mathrm{Ki} 67$ index were scanned at 20x magnification. A mask of tumor cells was semi-automatically created. Inside this mask blue (negative) and brown Ki67 positive nuclei were segmented using a Bayesian classifier. The Ki67 index was calculated using the areas of classified blue and brown nuclei. The square with the highest Ki67 index was used as the final result. A similar technique was used for $\mathrm{PPH} 3$. Not surprisingly, the reproducibility of the DAI-Ki67 and PPH3 counts by the automated digital image analysis on different days by different observers on 10 randomly selected cases was close to perfect $\left(\mathrm{R}^{2}=0.99\right)$.

\section{Data analysis}

For survival analysis, the main end points were distant metastases occurrence and overall distant metastases-related survival. To determine the probability that patients would remain free of distant metastases, we defined recurrence as any first recurrence at a distant site. Patients were censored from the date of the last follow-up visit for death from causes other than breast cancer, local or regional recurrences, and the development of a second primary cancer, including contralateral breast cancer. If a patient's status during follow-up indicated a confirmed metastasis without a re- 


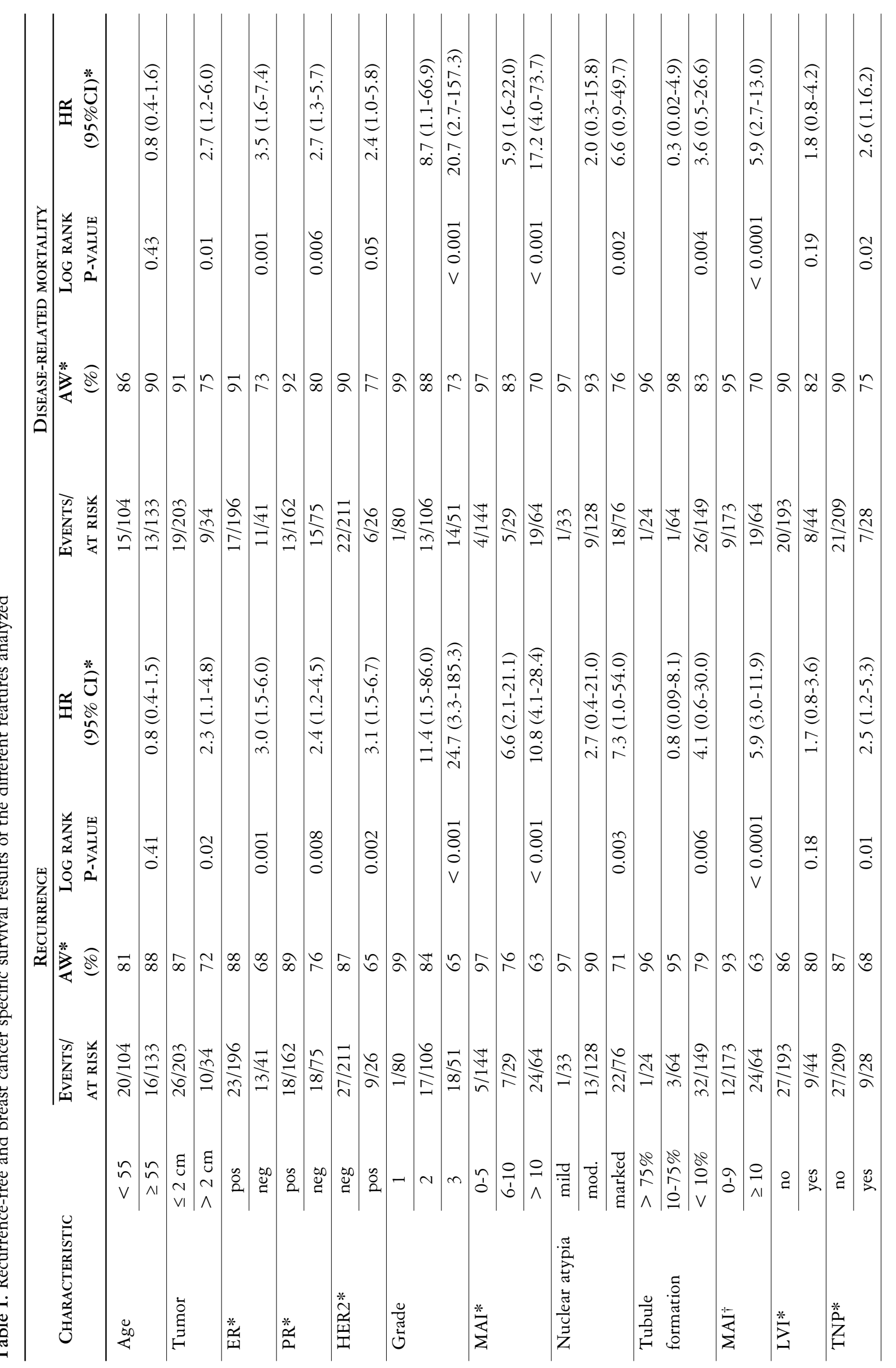




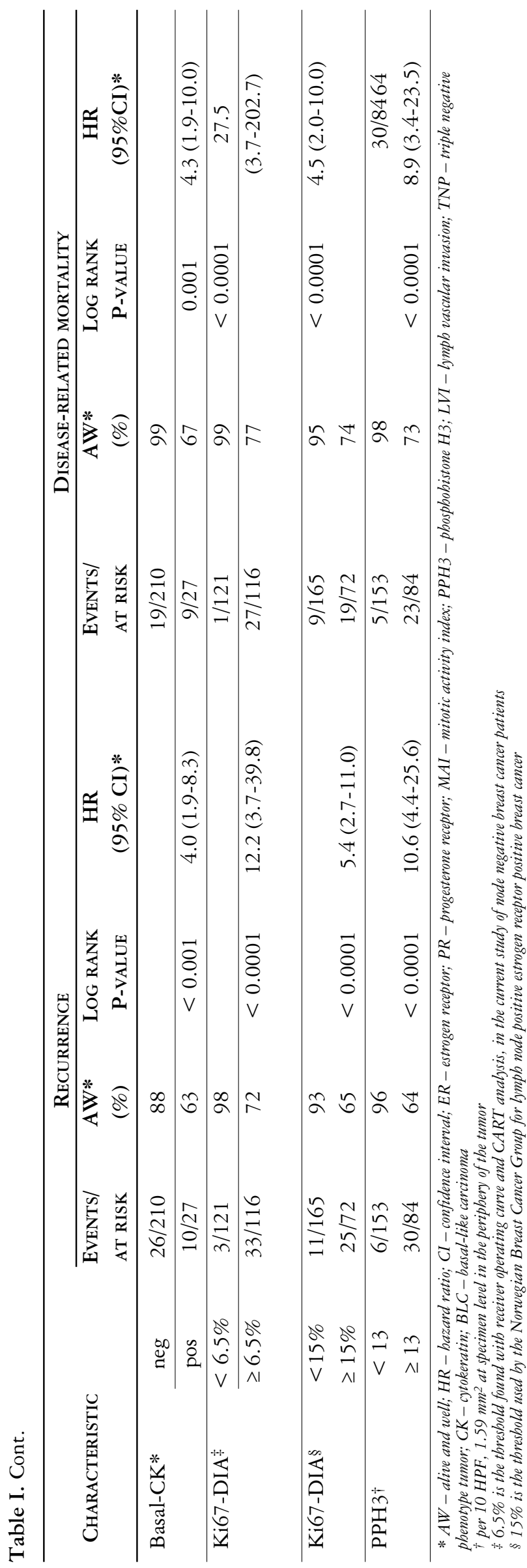

currence date, the follow-up visit date was used. Age, time to first recurrence, and survival time were calculated relative to the primary diagnosis date. For the MAI, three sets of previously established prognos-tic thresholds $[12](<6,6-10, \geq 11,<10$ versus $\geq 10$; and $<3,3-9$, and $\geq 10$ ) were examined. The prognostic thresholds were $6.5 \%$ for $\mathrm{Ki} 67$ and 13 per $1.59 \mathrm{~mm}^{2}$ at specimen level for PPH3. Kaplan-Meier survival curves were constructed, and between-group differences were tested using the log-rank test. The relative importance of potential prognostic variables was tested using Cox-proportional hazard analysis and expressed as a hazard ratio (HR) with a $95 \%$ confidence interval (CI).

\section{Results}

Thirty-six out of the 237 patients included in the study (15\%) developed distant metastases and $28(12 \%)$ died. Table I shows the univariate survival results.

With multivariate survival analysis, Ki67 prognostically overshadowed the following variables: age, tumor diameter, grade, ER, PR, HER2, CK5/6, CK14, triple negative phenotype tumor, basal-like cell type, lymph vessel invasion. $\mathrm{PPH} 3$ was however prognostically strongest, and DIA-Ki67-6.5\% added prognostically to $\mathrm{PPH} 3$ (Table II). The $\mathrm{PPH} 3 / \mathrm{Ki} 67$ combination therefore overshadowed all other features studied. Women with $\mathrm{PPH} 3<13$ and DIA-Ki67 $<6.5 \%$ have an excellent 10-year survival of $99 \%$, even without adjuvant systemic therapy. If $\mathrm{PPH} 3$ is $<13$, but DIA-Ki67 $\geq 6.5 \%$, the overall survival still is $90 \%$. When $\mathrm{PPH} 3 \geq 13$ the mortality is high even when $\mathrm{Ki} 67 \%$ is low (Table III). Table IV shows the prognostic interaction of MAI $<$ versus $\geq 10$, and DIA-Ki67$-6.5 \%$. In patients with MAI $<10$, DIA-Ki67 $<6.5 \%$ identifies a group with an excellent prognosis, but patients with $\mathrm{Ki} 67 \geq 6.5 \%$ have a significantly increased risk of dying from distant metastases $(\mathrm{p}=0.001$, hazard ratio $=14.8$ ). In patients with $\mathrm{MAI} \geq 10$, low Ki67 hardly occurs and has no additional prognostic value. $\mathrm{Ki} 67$ therefore is prognostically useful in patients with low proliferation according to either MAI or $\mathrm{PPH} 3$, but not in those with high MAI and $\mathrm{PPH} 3$ values.

\section{Discussion}

The current study shows that the proliferation features MAI, PPH3 and Ki67 (the latter two assessed by digital image processing) have strong prognostic value. $\mathrm{PPH} 3$ and MAI are strongly correlated, which is biologically understandable. DIA-Ki67 with a threshold of $6.5 \%$ is the strongest prognosticator of all $\mathrm{Ki} 67$ features, added prognostically to $\mathrm{PPH} 3$, and this combination overshadowed all other features studied.

As to the question why the proliferation markers MAI and PPH3 are prognostically stronger than Ki67, 
Table II. Multivariate comparison of all features shows that Ki67 by digital image analysis with a threshold of $6.5 \%$, in combination with $\mathrm{PPH} 3$ (with a threshold of 13) is the strongest prognostic combination explaining all other features

\begin{tabular}{|c|c|c|c|c|c|c|c|c|}
\hline \multicolumn{9}{|c|}{ VARIABLES IN THE EQUATION } \\
\hline & & \multirow[t]{4}{*}{ BETA } & \multirow{4}{*}{$\begin{array}{l}\text { STANDARD } \\
\text { ERROR }\end{array}$} & \multirow[t]{4}{*}{ WALD } & \multirow[t]{4}{*}{ SIGNIFICANCE } & \multirow{4}{*}{$\begin{array}{c}\text { HAZARD } \\
\text { RATIO }\end{array}$} & \multirow{3}{*}{\multicolumn{2}{|c|}{$\begin{array}{c}95 \% \text { CONFIDENCE } \\
\text { INTERVAL FOR } \\
\text { HAZARD RATIO } \\
\end{array}$}} \\
\hline & & & & & & & & \\
\hline & & & & & & & & \\
\hline & & & & & & & LOWER & UPPER \\
\hline Step 1 & $\begin{array}{c}\text { PPH3 }<13 \\
\text { vs. } \geq 13\end{array}$ & 2.2 & 0.49 & 19.6 & $<0.0001$ & 8.9 & 3.4 & 23.5 \\
\hline \multirow[t]{2}{*}{ Step 2} & $\begin{array}{c}\text { PPH } 3<13 \\
\text { vs. } \geq 13\end{array}$ & 1.2 & 0.52 & 4.9 & 0.03 & 3.2 & 1.1 & 8.9 \\
\hline & $\begin{array}{c}\text { DIA-Ki67 } \\
-6.5 \%\end{array}$ & 2.6 & 1.08 & 5.6 & 0.02 & 12.8 & 1.5 & 107.0 \\
\hline
\end{tabular}

VARIABLES NOT IN THE EQUATION IN STEP 2

\begin{tabular}{lc}
\hline FEATURE & $\begin{array}{c}\text { PROBABILITY } \\
\text { OF NO DIFFERENCE }\end{array}$ \\
\hline $\begin{array}{l}\text { age }<45,45-55,>55 \text { years } \\
\text { Tumor diameter } \\
\leq 2,>2 \mathrm{~cm}\end{array}$ & 0.59 \\
\hline $\begin{array}{l}\text { MAI } \\
0-2,>2\end{array}$ & 0.26 \\
\hline MAI & 0.51 \\
$0-9, \geq 10$ & 0.56 \\
\hline $\begin{array}{l}\text { MAI } \\
0-5,6-10,>10\end{array}$ & 0.50 \\
\hline grade & 0.23 \\
\hline estrogen receptor & 0.16 \\
\hline progesterone receptor & 0.87 \\
\hline HER2 negative, positive & 0.31 \\
\hline basal cell like negative, positive & 0.85 \\
\hline triple negative, positive & 0.29 \\
\hline cytokeratin 5/6 negative, positive & 0.13 \\
\hline cytokeratin 14 negative, positive & 0.73 \\
\hline lymph vessel invasion & \\
\hline
\end{tabular}

it is important to remember that $\mathrm{Ki} 67$ stains nuclei of cells in all phases of the cell cycle, i.e. G1-, S-, G2- and M-phase cells. However, many of these cells will go into the G0 phase or end in apoptosis as a result of DNA damage. In contrast, MAI exclusively identifies cells in the $\mathrm{M}$ phase and most of these cells will reach cell division. Likewise, $\mathrm{PPH} 3$-positive cells also have a much higher likelihood of dividing than $\mathrm{Ki} 67$ positive cells, as PPH3 stains only very late G2- and M-phase cells $[17,18]$.

The clinical use of the additional prognostic value of DIA-Ki67 in patients with $\mathrm{PPH} 3<13$ may depend on the attitude of the treating medical oncologist and the patient. Medical oncologists in the USA may re- gard a $10 \%$ risk of dying from metastatic disease too high to NOT give adjuvant systemic treatment. In north-west Europe, this risk is at the border of what often is regarded as just acceptable, as systemic chemotherapy in women $<55$ years old can have serious side effects.

Unfortunately, digital image analysis equipment is not yet widely available in pathology laboratories. This will most likely change in the years to come, with the advent of digital pathology. Until this has become a reality, pathologists could send their Ki67 stained sections to specialized laboratories which have the necessary computerized equipment. Alternatively, interactive morphometry assessment of Ki67 might be an inexpensive alternative [9]. Subjective counts not supported by point-weighted sampling, in our view, are not a defendable option as the determinations between pathologists vary too much.

In conclusion, in node negative breast cancer patients not undergoing adjuvant systemic treatment, $\mathrm{PPH} 3$ or MAI combined with $\mathrm{Ki} 67$ assessed by digital image analysis is prognostically strong, and therefore of potentially high clinical relevance.

None of the authors have any financial or other relationships with entities that have investment, licensing, or other commercial interests in the subject matter under consideration in this article.

\section{References}

1. Veronesi U, Boyle P, Goldhirsch A, et al. Breast cancer. Lancet 2005; 365: 1727-1741.

2. Adjuvant! Online, at http://www.adjuvantonline.com.

3. Boyages J, Chua B, Taylor R, et al. Use of the St Gallen classification for patients with node-negative breast cancer may lead to overuse of adjuvant chemotherapy. Br J Surg 2002; 89: 789-796.

4. Early Breast Cancer Trialists' Collaborative Group. Effects of chemotherapy and hormonal therapy for early breast cancer on recurrence and 15-year survival: an overview of the randomized trials. Lancet 2005; 365: 1687-1717. 
Table III. The additional prognostic value of DIA-Ki67-6.5\% is in the patients with a low PPH3 $(<13)$, not in the patients with $\mathrm{PPH} 3 \geq 13$

\begin{tabular}{|c|c|c|c|c|c|}
\hline \multicolumn{6}{|c|}{ CASE PROCESSING SUMMARY } \\
\hline \multirow[t]{2}{*}{ РPH3 } & \multirow[t]{2}{*}{ DIA-KI67 } & \multirow[t]{2}{*}{ Total N } & \multirow[t]{2}{*}{ N OF Events } & \multicolumn{2}{|c|}{ CENSORED } \\
\hline & & & & $\mathbf{N}$ & $\%$ \\
\hline \multirow{3}{*}{ PPH3 $<13$} & $<6.5 \%$ & 114 & 1 & 113 & 99.1 \\
\hline & $\geq 6.5 \%$ & 40 & 4 & 36 & 90.0 \\
\hline & overall & 154 & 5 & 149 & 96.8 \\
\hline \multirow{3}{*}{$\mathrm{PPH} 3 \geq 13$} & $<6.5 \%$ & 8 & 1 & 7 & 87.5 \\
\hline & $\geq 6.5 \%$ & 75 & 22 & 53 & 70.7 \\
\hline & overall & 83 & 23 & 60 & 72.3 \\
\hline overall & overall & 237 & 28 & 209 & 88.2 \\
\hline
\end{tabular}

\begin{tabular}{llccc}
\hline \multicolumn{5}{c}{ Overall comparisons } \\
\hline PPH3 $<13$ vs. $\geq 13$ & $\chi^{2}$ & DF & SIG. \\
\hline PPH3 $<13$ & Log Rank (Mantel-Cox) & 5.901 & 1 & 0.015 \\
\hline PPH3 $\geq 13$ & Log Rank (Mantel-Cox) & 0.707 & 1 & 0.400 \\
\hline
\end{tabular}

Test of equality of survival distributions for the different levels of DIA-Ki67 - 6.5\%.

Table IV. The additional prognostic value of DIA-Ki67-6.5\% is in patients with MAI $<10$

\begin{tabular}{|c|c|c|c|c|c|c|c|}
\hline \multicolumn{8}{|c|}{ CASE PROCESSING SUMMARY } \\
\hline \multicolumn{2}{|c|}{ MAI $<10$ vs. $\geq 10$} & \multicolumn{2}{|c|}{ KI67 BY DIA $6.45 \%$} & \multirow[t]{2}{*}{ TOTAL N } & \multirow[t]{2}{*}{$\mathbf{N}$ OF EVENTS } & \multicolumn{2}{|c|}{ CENSORED } \\
\hline & & & & & & $\mathbf{N}$ & $\%$ \\
\hline \multirow{7}{*}{ dimension 0} & & & $\mathrm{Ki} 67 \leq 6.45$ & 119 & 1 & 118 & 99.2 \\
\hline & $\mathrm{MAI}<10$ & dimension 1 & $\mathrm{Ki67}>6.45$ & 55 & 8 & 47 & 85.5 \\
\hline & & & overall & 174 & 9 & 165 & 94.8 \\
\hline & & & $\mathrm{Ki} 67 \leq 6.45$ & 2 & 0 & 2 & 100.0 \\
\hline & $\mathrm{MAI} \geq 10$ & dimension 1 & $\mathrm{Ki67}>6.45$ & 61 & 19 & 42 & 68.9 \\
\hline & & & overall & 63 & 19 & 44 & 69.8 \\
\hline & overall & dimension 1 & overall & 237 & 28 & 209 & 88.2 \\
\hline
\end{tabular}

\begin{tabular}{lccc}
\hline \multicolumn{4}{c}{ Overall comparisons } \\
\hline MAI $<10$ vs. $\geq 10$ & $\chi^{2}$ & DF & SIG. \\
\hline MAI $<10$ Log Rank (Mantel-Cox) & 10.9 & 1 & 0.001 \\
\hline MAI $\geq 10$ Log Rank (Mantel-Cox) & 0.60 & 1 & 0.44 \\
\hline
\end{tabular}

Test of equality of survival distributions for the different levels of Ki67 by DIA $6.45 \%$.

5. Baak JP, van Diest PJ, Voorhorst FJ, et al. Prospective multicenter validation of the independent prognostic value of the mitotic activity index in lymph node-negative breast cancer patients younger than 55 years. J Clin Oncol 2005; 23: 5993 6001.

6. Skaland I, Janssen EA, Gudlaugsson E, et al. Validating the prognostic value of proliferation measured by $\mathrm{PPH} 3$ (phosphohistone $\mathrm{H} 3$ ) in invasive lymph node-negative breast cancer patients less than 71 years of age. Breast Cancer Res Treat 2009; 115: 39-45.

7. Baak JP, Gudlaugsson E, Skaland I, et al. Proliferation is the strongest prognosticator in node-negative breast cancer: significance, error sources, alternatives and comparison with molecular prognostic markers. Breast Cancer Res Treat 2009; 115: 241-254.
8. Lende TH, Janssen EA, Gudlaugsson E, et al. In patients younger than age 55 years with lymph node-negative breast cancer, proliferation by mitotic activity index is prognostically superior to Adjuvant! J Clin Oncol 2011; 29: 852-858.

9. Gudlaugsson E, Skaland I, Janssen EA, et al. Comparison of the effect of different techniques for measurement of Ki67 proliferation on reproducibility and prognosis prediction accuracy in breast cancer. Histopathology 2012; 61: 1134-1144.

10. McShane LM, Atman DG, Sauerbrei W, et al. Reporting recommendations for tumor marker studies. J Clin Oncol 2005; 23: 9067-9072.

11. Pathology and Genetics: Tumors of the Breast and Female Genital Tract Organs; WHO Classification of Tumours Series. Tavassoli FA, Devilee P (eds.). IARC Press Lyon 2003; 9-112. 
12. Ellis IO, Schnitt SJ, Sastre-Garu X, et al. Invasive breast carcinoma. In: Tavassoli FA, Devilee P (eds). Pathology and Genetics of the Female Genital Tract Organs. IARC Press, Lyon 2003; 18-19.

13. van Diest PJ, Baak JP, Matze-Cok P, et al. Reproducibility of mitoses counting in 2469 breast cancer specimens: results from the Multicenter Morphometric Mammary Carcinoma Project. Hum Pathol 1992; 23: 603-607.

14. Jannink I, Risberg B, van Diest PJ, et al. Heterogeneity of mitotic activity in breast cancer. Histopatology 1996; 29: 421-428.

15. Bergers E, Jannink I, van Diest PJ, et al. The influence of fixation delay on mitotic activity and flow cytometric cell cycle variables. Hum Pathol 1997; 28: 95-100.

16. Gudlaugsson E, Skaland I, Undersrud E, et al. D2-40/p63 defined lymph vessel invasion has additional prognostic value in highly proliferating operable node negative breast cancer patients. Mod Pathol 2011; 24: 502-511.

17. Bossard C, Jarry A, Colombeix C, et al. Phosphohistone H3 labelling for histoprognostic grading of breast adenocarcinomas and computer assisted determination of mitotic index. J Clin Pathol 2006; 59: 706-710.

18. Skaland I, Janssen EA, Gudlaugsson E, et al. Phosphohistone $\mathrm{H} 3$ expression has much stronger prognostic value than classical prognosticators in invasive lymph node-negative breast cancer patients less than 55 years of age. Mod Pathol 2007; 12: 13071315 .

\section{Address for correspondence}

Jan P.A. Baak, MD, PhD, FRCPath(Hon), FICP(Hon)

Department of Pathology

Stavanger University Hospital

Armauer Hansen Rd 20

N-4068 Stavanger, Norway

tel. +4751519378

fax +4751519910

e-mail: jpabaak47@yahoo.com 